\title{
Free resolutions of certain codimension three perfect radical ideals
}

\author{
By \\ J. F. ANDrade ${ }^{1}$ ) and A. Simis ${ }^{1}$ )
}

\begin{abstract}
Introduction. The original conception of this work included a complete study of certain codimension three perfect ideals that arise naturally in a "determinantal" set-up, with the purpose of enriching the present collection of such ideals. The ones we consider are always radical and their syzygy-theoretic properties are relatively easy to describe. From the viewpoint of the theory of graded resolutions, they share common numerical features with (the projecting cones of) certain codimension two and three varieties in projective space. Their resolutions being seldom pure, they seemed largely convenient for testing the sharpness of some calculations made in the pure case (cf. [19], [25], [26]). Moreover, they yield a large class suited for testing recently developed concepts, such as the notion of strong obstruction, that have its natural place in the theory of linkage ([loc. cit.]) or the more general theory of residual intersection developed in [16]. The authors did not pursue this line of thought, but it seemed worthwhile pointing it out.
\end{abstract}

A brief description of the present contents is as follows. In the first section one obtains the explicit free resolution of a large class of codimension three ideals that appear as presentation ideals of normal cones associated to determinantal ideals fixing a submatrix. As it turns out, the resolutions of these presentation ideals are given by a mapping cylinder of a suitable map to the resolution of the original determinantal ideals.

The second section is concerned with deriving some ideal-theoretic results from the knowledge of the presentation given in section one. This procedure, by and large, follows the techniques developed by various authors ([17], [14], [23] and [24]).

In the third section one gives the resolution of the determinantal ideal $J_{r}$ associated to a map $R^{m} \rightarrow R^{n}(m \geqq n)$, fixing $R^{r} \subset R^{m}$, in the case of the data $r=n-2$ and $m=n+2$. This case not only extends the previous known ones [1], [2], but mainly gives some genuine clue for the general situation. The construction follows closely the spirit of the "scandinavian" complex [9] in that one is led to tensor suitable complexes and then to cut down the resulting size by an use of "trace" maps. This method seems to generalize well in various contexts [21].

Last one establishes exact values for the codimension of the complete intersection locus of the ideals considered in the previous sections. The reason for doing so is that the known estimates to present [11], [27] would give no real grasp, in this case, of the locus.

1) Both authors were partially supported by CNPq (Brazil) 
1. Free resolutions of normal cones associated to determinantal loci fixing a submatrix. Let $(\boldsymbol{X})$ be an $n \times m(n \leqq m)$ matrix of indeterminates over a field $k$. Set $R:=k[X]$ and let $J_{r}=J_{r}(X)$ stand for the ideal of $R$ generated by the minors of $(X)$ that fix the first $r$ columns. A thorough study of $J_{r}$ was the theme of [2], while a detailed account of the properties of the Rees algebra $R\left(J_{r}\right)$ and of the associated graded ring $\operatorname{gr}_{J_{r}}(R)$, in the cases $r=n-1$ and $m=n+1$, was provided in [6]. Thus, for instance, if $r=n-1, R\left(J_{r}\right)$ is given by the determinantal ring of maximal minors of the matrix

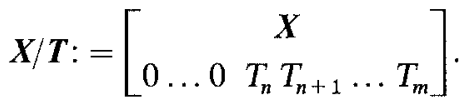

From this one sees that the Eagon-Northcott complex yields a free resolution of $R\left(J_{r}\right)$ over the polynomial ring $R[T]$. The resolution of $\operatorname{gr}_{J_{r}}(R)$ over $R[T]$ is a little harder.

In this section one sticks to the case where $r=n-1$ and $m=n+2$ both because of its simplicity and the fact that the presentation ideal over $R[T]$ has codimension three. On the other hand, the original set-up will be slightly extended. Namely, let $R$ be a noetherian ring, let $f: R^{n+2} \rightarrow R^{n}$ be a map and let there be given a splitting $R^{n+2}=R^{n-1} \oplus R^{3}$. Let $f^{\prime}$ stand for the restriction of $f$ to $R^{n-1}$ and let $J_{n-1}=J_{n-1}(f) \subset A^{n} R^{n}=R$ denote the image of the map $A^{n} f$ restricted to the module $A^{n-1} R^{n-1} \otimes A^{1} R^{3}$.

According to [1], if grade $\left(I_{n-1}\left(f^{\prime}\right)\right) \geqq 2$ and grade $\left(I_{n}(f)\right) \geqq 3$ then $R / J_{n-1}$ admits a finite free resolution:

$$
\mathscr{F}: 0 \longrightarrow A^{n+2} R^{n+2} \otimes S_{1}\left(R^{n}\right) \stackrel{\varphi_{3}}{\longrightarrow} A^{n+1} R^{n+2} \stackrel{\varphi_{2}}{\longrightarrow} R^{3} \stackrel{\varphi_{1}}{\longrightarrow} R,
$$

with the identifications $A^{n-1} R^{n-1} \otimes \Lambda^{1} R^{3}=R^{3}$ and $\varphi_{1}=A^{n} f$ restricted to $R^{3}$.

One also has the acyclic (provided grade $\left(I_{n-1}\left(f^{\prime}\right)\right) \geqq 2$ ) complex

$$
\mathscr{G}^{*}: 0 \longrightarrow R \stackrel{\delta}{\longrightarrow}: R^{n^{*}} \stackrel{f^{\prime *}}{\longrightarrow} R^{n-1 *}
$$

where $\delta=\left(A^{n-1} f^{\prime *}\right)=A^{n-1} f^{\prime}, R^{n^{*}}=A^{n-1} R^{n}, R=A^{n-1} R^{n-1}$.

The maps $\varphi_{3}$ and $\varphi_{2}$ are easy to describe and one refers to [1] for the details. The exact knowledge of $\varphi_{3}$ and $\varphi_{2}$ are inessential for the construction we are about to consider it suffices to realize that the restriction of $\varphi_{2}$ to $A^{n-1} R^{n-1} \otimes A^{2} R^{3} \subset A^{n+1} R^{n+2}$ is given by the Koszul map $A^{2} R^{3} \rightarrow R^{3}$ on the three generators of $J_{n-1}$.

Next, let $T:=T_{n}, T_{n+1}, T_{n+2}$ be a set of three indeterminates over $R$, so numbered for convenience. Set $B:=R[T]$. We will define a (degree preserving) map of complexes $\eta: \mathscr{G} \otimes_{R} B \rightarrow \mathscr{F} \otimes_{R} B$. For this, one uses the Koszul complex on $T$, whose differentials will be denoted $\chi_{i}(i=1,2,3)$, and the map

$$
f / T:=\left[\begin{array}{cc}
f \\
0 \ldots 0 & \chi_{1}
\end{array}\right]: B^{n-1} \oplus B^{3} \rightarrow B^{n} \oplus B .
$$

We will also use the following identifications in addition to the above:

$$
\begin{aligned}
\left(B^{n-1}\right)^{*} & =A^{n-2} B^{n-1} \\
& =\left(A^{n-2} B^{n-1} \otimes A^{3} B^{3}\right) \otimes A^{n+1} B^{n+1} \subset A^{n+1} B^{n+2} \otimes A^{n+1} B^{n+1} \\
\left(B^{3}\right)^{*} & =A^{2} B^{3} ; \quad B=A^{n-1} B^{n-1} ; \quad A^{2} B^{3}=A^{n-1} B^{n-1} \otimes A^{2} B^{3} .
\end{aligned}
$$


One is now ready for the definition of the map $\eta$, which is very simple. Namely, set

$$
\begin{aligned}
& \eta_{0}:=\text { restriction of } A^{n+1}(f / T) \text { to }\left(B^{n-1}\right)^{*} \\
& \eta_{1}:=\varkappa_{2}{ }^{\circ} I_{2}^{*} \circ\left(f^{*} \otimes B\right), \text { where } l_{2}: B^{3} \rightarrow B^{n+2} \text { is the inclusion } \\
& \eta_{2}:=x_{3}
\end{aligned}
$$

Here is the main result of this section:

Proposition 1.1 (i) $\eta: \mathscr{G} \otimes_{R} B \rightarrow \mathscr{F} \otimes_{R} B$ is a map of complexes.

(ii) If grade $\left(I_{n-1}\left(f^{\prime}\right)\right) \geqq 2$ and grade $\left(I_{n}(f)\right) \geqq 3$, then the mapping cylinder $C(\eta)$ is a free B-resolution of $S\left(J_{n-1} / J_{n-1}^{2}\right)$, the symmetric algebra of the conormal module of $J_{n-1}$.

Proof. First we show that $\eta$ is indeed a map of complexes, i.e., that

$$
\left.A^{n} f \otimes B\right|_{B^{3}} \circ x_{2} \circ I_{2}^{*}\left(f^{*} \otimes B\right)=\left.\left.\left(A^{n+1} f / T\right)\right|_{\left(B^{n-1}\right)^{*}} \circ(f \otimes B)\right|_{B^{n-1}} ^{*}
$$

and

$$
\left.\varphi_{2} \otimes B\right|_{\left(A^{n-1} B^{n-1} \otimes A^{2} B^{3}\right)} \circ \chi_{3}=\chi_{2} \circ l_{2}^{*} \circ\left(f^{*} \otimes B\right) \circ(\delta \otimes B) .
$$

Now, (1) is just a fancy way of writing the Cramer-Laplace relations for the $(n+1) \times(n+1)$ minors of the map $f / T$, along its rows. As to (2), it expresses the elementary fact to the effect that, in order to write down the $(n+1) \times(n+1)$ minors of $f / T$ fixing the first $n-1$ columns, one can first expand by Laplace along the $(n+1)$-th row and then expand the corresponding cofactors (= generators of $J_{n-1}$ ) along the column complementary to the first $n-1$ columns. Incidentally, of course, (1) and (2) are also expressions of higher order Laplace relations or expansions.

Next, since $\mathscr{G} \otimes B$ and $\mathscr{F} \otimes B$ are cyclic under the given hypotheses, it follows that the mapping cylinder $C(\eta)$ is also acyclic. Therefore, $C(\eta)$ is a free $B$-resolution of coker $\left(B^{3} \oplus\left(B^{n-1}\right)^{*} \underset{\left(\varphi_{1} \otimes B, \eta_{0}\right)}{\longrightarrow} B\right)$. An easy inspection shows that this cokernel is isomorphic to $S\left(J_{n-1} / J_{n-1}^{2}\right)$.

Corollary 1.2. Let $f: R^{n+2} \rightarrow R^{n}$ be a generic (say, over a field) map. Then $C(\eta)$ is a free $B$-resolution of $\operatorname{gr}_{J_{n-1}}(R)$.

Pro of. By [2] (cf. also [6]), $J_{n-1}$ is of linear type, i. e., its symmetric and Rees algebras coincide.

R e m a rk. As a graded $B$-module - where $B$ is graded in the usual way, $R$ being its piece of degree zero $-S\left(J_{n-1} / J_{n-1}^{2}\right)$ admits a graded resolution

$$
0 \rightarrow B^{n} \oplus B(-3) \rightarrow B^{n+2} \oplus B(-2)^{n} \rightarrow B^{3} \oplus B(-1)^{n-1} \rightarrow B .
$$

Perhaps more interesting is the generic case: one regrades $B$ so as to have the entries of the map $f$ of degree one. Then $S\left(J / J^{2}\right)=\operatorname{gr}_{J}(R)$ admits the graded homogeneous resolution

$$
\begin{aligned}
0 & \rightarrow B(-(2 n+1))^{n+1} \rightarrow B(-2 n)^{n+2} \oplus B(-(n+2))^{n} \\
& \rightarrow B(-n)^{3^{*} \oplus B(-(n+1))^{n-1} \rightarrow B} .
\end{aligned}
$$


The case $n=2$ gives a presentation ideal $I \subset B$ which is an almost complete intersection. Moreover, $I$ admits a minimal $B$-sequence which is part of a minimal set of generators. Therefore, $I$ is linked to a Gorenstein ideal, necessarily generated by the Pfaffians of a skew-symmetric matrix over $B$ [4]. This ideal was explicitly given in [22] in order to test a formula for the multiplicity of $B / I$. For $n \geqq 4, I$ has deviation $n+2-3=n-1 \geqq 3$. It seems natural to ask whether $I$ is strongly obstructed - i.e., whether the twisted conormal module $w_{B / I} \otimes I$ is Cohen-Macaulay - or, even more, whether $I$ is in the linkage class of a complete intersection.

One even lacks the information as to whether the first Koszul homology module on the generators of $I$ is Cohen-Macaulay - a negative answer to this would discard any hopes to extending Vasconcelos conjecture (A) [26] beyond the realm of pure resolutions.

2. Analytic behaviour of the normal cone. The following generality will serve our purpose in this section: $B$ is a normal quasi-unmixed domain and $I \subset B$ is an ideal of finite homological dimension and codimension at least two.

We recall that, quite generally, for a normal domain $B$ and an ideal $I \subset B$ of codimension at least two, such that the Rees algebra $R(I)$ is normal, there is the so called exact sequence of divisor class groups [23], [18]:

$$
0 \rightarrow \mathbb{Z}^{r} \rightarrow \mathrm{Cl}(R(I)) \rightarrow \mathrm{Cl}(B) \rightarrow 0,
$$

where $r$ is the number of height one primes of the exceptional divisor $I R(I)$.

As a notation, $v(E)$ will stand for the minimal number of generators of a finitely generated module over a local ring.

For the reader's convenience, we collect out of various sources the pertinent results.

Theorem 2.1. Let $B$ be a normal quasi-unmixed domain and let $I \subset B$ be a radical ideal of codimension at least two and finite homological dimension. If $I$ is of linear type then the following conditions are equivalent:

(i) The symmetric algebra $S(I)$ is a normal domain and $\operatorname{ker}(\mathrm{Cl}(S(I)) \rightarrow \mathrm{Cl}(B))$ is (a free group) of rank equal to the number of associated primes of $B / I$.

(ii) $\operatorname{gr}_{I}(R)$ is $R / I$-torsion free.

(iii) $\operatorname{gr}_{I}(R)$ is reduced.

(iv) $v\left(I_{p}\right) \leqq \max \left\{\mathrm{ht}\left(I_{p}\right)\right.$, ht $\left.(P)-1\right\}$, for every prime $P \supset I$.

(v) For a presentation $F_{2} \stackrel{\psi}{\longrightarrow} F_{1} \longrightarrow I \longrightarrow 0$, one has ht $\left(I_{t}(\psi)\right) \geqq \operatorname{rk}(\psi)-t+3$, for $1 \leqq t \leqq \operatorname{dev}(I):=\operatorname{rk}\left(F_{1}\right)-\operatorname{ht}(I)$.

P r o o f. The equivalences (i) $\Leftrightarrow$ (ii) $\Leftrightarrow$ (iii) are in [17] (cf. also [23] for further generality in (i) $\Leftrightarrow$ (ii)). We refer to [14] for the equivalences (ii) $\Leftrightarrow$ (iv) $\Leftrightarrow$ (v).

Lemma 2.2. Let $I \subset B:=R[\boldsymbol{T}], R=k[X]$, stand for the presentation ideal of the ring $\operatorname{gr}_{J_{n-1}}(R)$ as in Section 1 (generic case). Then $v\left(I_{p}\right) \leqq \max \left\{\mathrm{ht}\left(I_{p}\right)\right.$, ht $\left.(P)-1\right\}$ for every $P \supset I$. 
Proof. By the results of Section 1 as applied in this case, one has a minimal presentation

$$
\begin{aligned}
& B^{2 n+2}=B^{n+2} \oplus B^{n} \stackrel{\psi}{\longrightarrow} B^{n+2}=B^{3} \oplus B^{n-1} \longrightarrow I \longrightarrow 0, \\
& \psi=\left[\begin{array}{c|c}
\varphi_{2} \otimes B & \eta_{1} \\
\hline 0 & \left(f^{\prime} \otimes B\right)^{*}
\end{array}\right], \quad f^{\prime}:=\left.f\right|_{R^{n-1}} .
\end{aligned}
$$

First observe that $v(I)=n+2$, so one may assume that ht $(P) \leqq n+2$. Induct on $n$. Let $n \geqq 3$. Then ht $\left(I_{1}\left(f^{\prime} \otimes B\right)^{*}\right)=n(n-1)>n+2$, hence, say, $X_{11} \notin P$. By inverting this element and performing elementary transformations on the matrix $X / T$, one obtains a matrix of the same form on in new indeterminates $Y_{i j}=X_{i j}-X_{1 i} X_{i 1} X_{11}^{-1}$ in a new ring $\widetilde{B} \cong B\left[X_{11}^{-1}\right]$ and, moreover, $I\left[X_{11}^{-1}\right] \cong \widetilde{I} \subset \widetilde{B}$, where $\widetilde{I}$ is the ideal of maximal minors of the new matrix. Also, the ring $\operatorname{gr}_{\widetilde{J}_{n-1}}(\tilde{R})$ is presented over $\widetilde{B}$ by $\tilde{I}$, where $\tilde{J}_{n-1} \subset \widetilde{R} \cong R\left[X_{11}^{-1}\right]$ is the corresponding ideal of maximal minors of $(Y)$ fixing the initial $(n-1) \times(n-2)$ submatrix. Letting $\widetilde{P}:=P \widetilde{B}$, we have $I_{P} \cong \widetilde{I}_{\widetilde{P}}$ and ht $(P)=\operatorname{ht}(\widetilde{P})$.

In this way, we have reduced the entire argument to the case $n=2$. Here, we are assuming that ht $(P)=4$. Looking closer at the presentation of $I$, one sees that $I_{1}\left(\varphi_{2} \otimes B \mid \eta_{1}\right)$ contains the cofactors of the $3 \times 3$ matrix

$$
\left[\begin{array}{ccc}
X_{12} & X_{13} & X_{14} \\
X_{22} & X_{23} & X_{24} \\
T_{2} & T_{3} & T_{4}
\end{array}\right] .
$$

Further, $I_{1}\left(f^{\prime} \otimes B\right)^{*}=\left(X_{11}, X_{21}\right) B$, a regular sequence modulo the previous cofactors. Therefore, one can invert a coefficient of some relation among the generators of $I$. This shows our contention.

Corollary 2.3. Keeping the hypotheses of Lemma 2.2, if $n=2,3$ then $S(I)$ is normal and $\mathrm{Cl}(S(I)) \cong \mathbb{Z}^{2}$.

Proof. For $n=2,3, I$ has deviation at most 2, hence is strongly Cohen-Macaulay [26]. Applying the above lemma, it follows that $I$ is of linear type [14]. The result now follows from Proposition 2.1.

R e $\mathrm{m}$ a rk. Even if $I$ happens not to be of linear type, one can still derive similar results for the Rees algebra. However, the authors expect Corollary 2.3 to be valid for any value of $n$. A question remains as to whether one can prescribe, in the non-generic case, sufficient estimates for the grades of the Fitting ideals in order to obtain results along the same line.

3. The free resolution of the maximal minors fixing a submatrix: the unmixed case. Referring to the notation of Section 1, recall that $J_{r}=J_{r}(f)$ stands for the ideal $\left(A^{n} f\right)\left(A^{r} R^{r} \otimes A^{n-r} R^{m-r}\right) \subset R$, where $f: R^{m} \rightarrow R^{n}$ is a map and there is given a splitting $R^{m}=R^{r} \oplus R^{m-r}(m \geqq n+1, n \geqq r+1)$. If $f$ is "generic" then one of the results of [2] is to the effect that the codimension of $J_{r}$ equals the minimum value between $m-n+1$ and $n-r+1$, while the homological dimension of $R / J_{r}$ is $m-r$.

In general, one would like to prove:

Conjecture. (R noetherian) If grade $I_{n}(f) \geqq m-n+1$, grade $I_{r}\left(f \mid R^{r}\right) \geqq n-r+1$ and if $I_{n}(f)+I_{n-2}\left(\left.f\right|_{R^{r}}\right)$ is a proper ideal of grade at least $m-r$, then $R / J_{r}$ has homological dimension $m-r$. 
We observe that in earlier works [1], [2] the third grade requirement was automatically subsumed in the first two. In general, however, this additional bound is essential, as the following example shows: let $R=k[X, Y, Z]$ and let $f$ be represented by the $3 \times 5$ matrix, where we fix the first column:

$$
M:=\left[\begin{array}{ccccc}
X & Y & Z & 0 & 0 \\
Y & X & Y & Z & 0 \\
Z & 0 & X & Y & Z
\end{array}\right] .
$$

Here, $I_{1}\left(M^{\prime}\right)=(X, Y, Z)$ and $I_{3}(M)=(X, Y, Z)^{3}$ have grade three, but of course, the homological dimension of any $R$-module is at most three, whereas $5-1=4$.

Clearly, one would like to conjecture about the actual structure of a finite free resolution of $J_{r}$ under the above conditions. Once an explicit presentation of $J_{r}$ is at hand, finding the codimension of $J_{r}$ is, in principle, an easy task. A good candidate is given by a variation of the Cramer map (cf. the proof of Theorem 3.1). It is much harder to guess the remaining maps of a (potential) free resolution. The authors believe that there is such a resolution obtained by tensoring well-known free complexes and by cutting the faulty sizes by suitable trace maps.

In this section, we illustrate this expectation in a particular case which, nevertheless, is believed to keep the main features of the general unmixed case, that is to say, the case where $m-n+1=n-r+1$. The reason we focus on unmixed ideals, at this stage, is that, among other nice properties, they enjoy the (rare?) phenomenon that their associated graded ring is Gorenstein while not being a domain (a general account of such phenomena is to be found in [15]).

First, one has the well-known Buchsbaum-Rim complexes that, under the present hypotheses, resolve the cokernel of the maps $R^{n+2} \stackrel{f}{\rightarrow} R^{n}$ and $\left(R^{n}\right)^{*} \stackrel{f^{\prime *}}{\rightarrow}\left(R^{n-2}\right)^{*}$, respectively (cf. [5]). We are particularly interested in the tail maps of these complexes, namely, $0 \rightarrow R^{n} \rightarrow A^{n+1} R^{n+2}$ and $0 \rightarrow\left(R^{n-2}\right)^{*} \rightarrow A^{n-1}\left(R^{n}\right)^{*}$. The tensor product of these maps yields a complex

$$
\begin{aligned}
0 \rightarrow R^{n} \otimes\left(R^{n-2}\right)^{*} \stackrel{\varphi_{4}}{\longrightarrow}\left(R^{n} \otimes A^{n-1}\left(R^{n}\right)^{*}\right) \\
\\
\oplus\left(\left(R^{n-2}\right)^{*} \otimes A^{n+1} R^{n+2}\right) \stackrel{\varphi_{3}}{\longrightarrow} A^{n+1} R^{n+2} \otimes A^{n-1}\left(R^{n}\right)^{*}
\end{aligned}
$$

which we modify as follows: project the module $A^{n+1} R^{n+2}$ onto its direct summand $A^{n-3} R^{n-2} \otimes A^{4} R^{4}$ and compose with the trace map $(V, W) \rightarrow \operatorname{tr} V+\operatorname{tr} W$, where the module $R^{n} \otimes A^{n-1}\left(R^{n}\right)^{*}$ (respectively, $\left(R^{n-2}\right)^{*} \otimes A^{n-3} R^{n-2}$ ) is identified with the free $R$-module whose elements are the $n \times n$ (respectively, $(n-2) \times(n-2))$ matrices over $R$. The result of this composition clearly maps onto $R$, hence its kernel $K$ is a free $R$-module of rank $2 n^{2}-5$. It is easy to see that $\operatorname{im}\left(\varphi_{4}\right) \subset K$.

This takes care of the "trace" modification of the tensor product of the maps. We further introduce the following variation of the "Cramer map" associated to the map $f: R^{n+2} \rightarrow R^{n}:$

$$
\begin{aligned}
& A^{n+1} R^{n+2} \otimes\left(A^{n-1} R^{n}\right)^{*} \rightarrow A^{2} R^{n+2} \\
& w_{1} A \ldots A w_{n+1} \otimes \Phi \mapsto \sum_{1 \leqq j_{1} \leqq j_{2} \leqq n+1} \sigma\left(j_{1}, j_{2}\right) \Phi\left(f\left(w_{i_{1}}\right) A \ldots A f\left(w_{i_{n-1}}\right)\right) w_{j_{1}} A w_{j_{2}},
\end{aligned}
$$


where $\sigma\left(j_{1}, j_{2}\right)$ is the sign of the permutation $1 \rightarrow i_{1}, \ldots, n \rightarrow j_{1}, n+1 \rightarrow j_{2}$, with $\{1, \ldots, n+1\}=\left\{i_{1}, \ldots, i_{n-1}, j_{1}, j_{2}\right\}$.

Composing this map with the canonical projection of $A^{2} R^{n+2}$ onto its summand $A^{2} R^{4} \cong A^{n-2} R^{n-2} \otimes A^{2} R^{4}$, we obtain a map $\varphi_{2}$. Finally, set $\varphi_{1}:=$ restriction of $A^{n} f$ to $A^{n-2} R^{n-2} \otimes A^{2} R^{4}$.

Lemma 3.1. The above construction yields a complex

$$
\begin{aligned}
\mathscr{C}: 0 \longrightarrow R^{n} \otimes\left(R^{n-2}\right)^{*} \stackrel{\varphi_{4}}{\longrightarrow} K \stackrel{\left.\varphi_{3}\right|_{K}}{\longrightarrow} A^{n+1} R^{n+2} \\
\otimes\left(A^{n-1} R^{n}\right)^{*} \stackrel{\varphi_{2}}{\longrightarrow} \Lambda^{n-2} R^{n-2} \otimes \Lambda^{2} R^{4} \stackrel{\varphi_{1}}{\longrightarrow} R .
\end{aligned}
$$

Proof. We are to verify that $\left.\varphi_{2} \circ \varphi_{3}\right|_{K}$ and $\varphi_{1}{ }^{\circ} \varphi_{2}$ are the zero maps. The fact that $\varphi_{1}{ }^{\circ} \varphi_{2}=0$ is a base-free restatement of the straightening relations of the $n \times n$ minors of $f$ fixing the submodule $R^{n-2} \subset R^{n+2}$. As for the other composition, the argument goes as follows. Choose bases $f_{1}, \ldots, f_{n}$ and $e_{1}, \ldots, e_{n+2}$ of $R^{n}$ and $R^{n+2}$, respectively. Then, a basis of $K$ is given by the following collection of elements:

(1) $f_{i} \otimes f_{1}^{*} A \ldots A \widehat{f}_{l}^{*} \Lambda \ldots A f_{n}^{*}, \quad i \neq l, \quad 1 \leqq i, l \leqq n$

(2) $e_{j}^{*} \otimes e_{1} A \ldots A \hat{e}_{k} A \ldots A e_{n+2}, \quad j \neq k, \quad 1 \leqq j \leqq n-2, \quad 1 \leqq k \leqq n+2$

(3) $f_{i} \otimes f_{1}^{*} \Lambda \ldots \Lambda \hat{f}_{i}^{*} \Lambda \ldots \Lambda f_{n}^{*}-f_{1} \otimes \hat{f}_{1}^{*} \Lambda f_{2}^{*} \Lambda \ldots \Lambda f_{n}^{*}, \quad i \neq 1, \quad 1 \leqq i \leqq n$

(4) $e_{j}^{*} \otimes e_{1} A \ldots A \hat{e}_{j} A \ldots A e_{n+2}-f_{1} \otimes \hat{f}_{1}^{*} A f_{2}^{*} A \ldots \Lambda f_{n}^{*}, \quad 1 \leqq j \leqq n-2$.

To prove our contention, we compute the value of $\varphi_{2} \circ \varphi_{3}$ on each class as above of basis elements. Leaving out the details of the computation for space reasons, we obtain that the vanishing of $\varphi_{2} \circ \varphi_{3}$ on class (1) expresses the Cramer-Laplace relations of the $(m-1) \times(m-1)$ minors of an $(m-1) \times m$ submatrix of $f$ with rows $1, \ldots, \hat{l}, \ldots, n$, along the $i$-th row $(i \neq l)$; on elements of class (2), it expresses the same provided "row" is changed to "column". On class (3), the vanishing of $\varphi_{2} \circ \varphi_{3}$ translates into the fact that an $n \times n$ minor of $f$ can be developed along the $i$-th row $(i \neq 1)$ or along the first row. A similar translation applies in the case of class (4).

Lemma 3.2. If grade $I_{n}(f) \geqq 3$, grade $I_{n-2}\left(f_{\left.\right|_{R^{n-2}}}\right) \geqq 3$ and grade $\left(I_{n}(f)+I_{n-2}\left(f_{\left.\right|_{R} ^{n-2}}\right)\right)$ $\geqq 4$, then the complex $\mathscr{C}$ is acyclic.

Proof. By the "lemme d'acyclicite" [20], it suffices to establish the acyclicity of $\mathscr{C}_{P}$ for a prime ideal $P \subset R$ of height at most three. For such a prime, by hypothesis, we must have either $I_{n}(f) \notin P$ or else $I_{n-2}\left(f^{\prime}\right) \notin P$ (with $f^{\prime}=f_{\left.\right|_{R^{n-2}}}$ ).

Let $I_{n}(f) \notin P$ and let $\Delta$ denote an $n \times n$ minor not lying in $P$. If such a minor involves the first $n-2$ columns of $f$, then $\left(\varphi_{1}\right)_{P}$ splits and, therefore, $\mathscr{C}_{P}$ is split exact all the way through. Thus, we assume $A$ does not involve all of the first $n-2$ columns of $f^{\prime}$. In this case, by applying suitable elementary column transformations on $f$ that do not affect the ideals im $\left(\varphi_{1}\right)$ and $I_{n-2}\left(f^{\prime}\right)$, one can assume that $\Delta$ is the $n \times n$ minor with columns $2, \ldots, n+1$ or the one with columns $3, \ldots, n+2$. Then, by inverting $\Delta$ (in $R_{P}$ ) and applying further elementary transformations, one can represent $f_{P}$, respectively, by the matrices

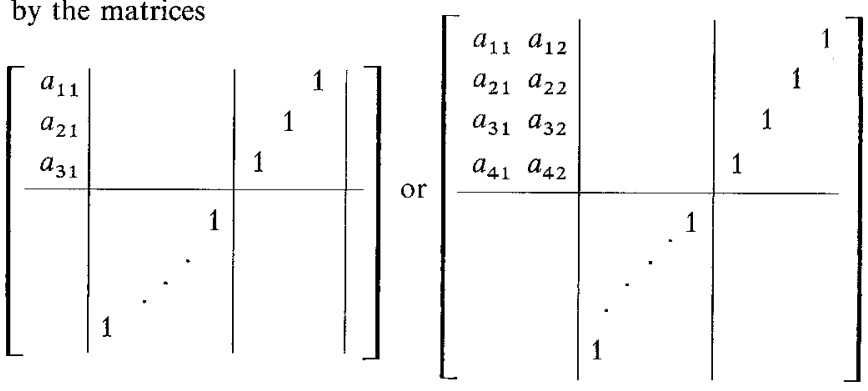


where all unspecified entries are zero. Next, a convenient choice of bases sets the complex $\mathscr{C}_{P}$ in a form suitable for applying the criterion of Buchsbaum-Eisenbud [3]. Thus, if $I(\psi)$ denotes the ideal of $(\mathrm{rk} \psi) \times(\mathrm{rk} \psi)$ minors of a matrix $\psi$, one obtains in both cases that, up to radical, $I\left(\varphi_{i}\right)_{P}$ and $I_{n-2}\left(f_{P}^{\prime}\right)$ are one and the same ideal for $1 \leqq i \leqq 3$ and $I\left(\varphi_{4}\right)_{P}=R_{P}$. Therefore, $\mathscr{C}_{P}$ is acyclic.

Let now $I_{n-2}\left(f^{\prime}\right) \notin P$. Assume, as we may, that the $(n-2) \times(n-2)$ minor of $f$ with rows $1, \ldots, n-2$ and columns $1, \ldots, n-2$ does not belong to $P$. By a similar token, the matrix of $f_{P}$ can be set in the form

$$
\left[\begin{array}{l|llll}
I_{n-2} & & & & \\
\hline & a_{n-1, n-1} & a_{n-1, n} & a_{n-1, n+1} & a_{n-1, n+2} \\
& a_{n, n-1} & a_{n, n} & a_{n, n+1} & a_{n, n+2}
\end{array}\right] .
$$

Again, by computing the depths of $I\left(\varphi_{i}\right)_{P}$, one sees that $\mathscr{C}_{P}$ is acyclic.

The main theorem of this section can be stated as follows.

Theorem 3.3. The following conditions are equivalent:

(i) grade $I_{n}(f) \geqq 3$, grade $I_{n-2}\left(\left.f\right|_{R^{n-2}}\right) \geqq 3$ and grade $\left(I_{n}(f)+I_{n-2}\left(\left.f\right|_{R^{n-2}}\right)\right) \geqq 4$.

(ii) The complex $\mathscr{C}$ is acyclic.

Moreover, in that case, the homological dimension of $R / J_{n-2}(f)$ is exactly four if and only if the ideal $I_{n}(f)+I_{n-2}\left(\left.f\right|_{R^{n-2}}\right)$ is proper.

P r o of. Lemma 3.2 above takes care of the implication (i) $\Rightarrow$ (ii). To prove the converse implication we argue as follows. Let $\tilde{f}$ be the corresponding generic map whose entries are indeterminates over $R$; let $\tilde{f}^{\prime}, \tilde{J}$, etc. stand for the corresponding data. Since the conditions in (i) are well-known to be satisfied in this generic situation, the corresponding complex $\widetilde{\mathscr{C}}$ is acyclic by the first implication and, moreover, grade $\widetilde{J}=3$ as $\widetilde{J}=I_{n}(\tilde{f}) \cap I_{n-2}\left(\tilde{f}^{\prime}\right)$. This shows easily that we have equality, up to radicals, of the ideals $I\left(\tilde{\varphi}_{3}\right)$ and $I\left(\tilde{\varphi}_{2}\right)$ respectively taken "at the rank" of the matrices). By specialization, we have at least $I\left(\varphi_{3}\right) \subset \sqrt{I\left(\varphi_{2}\right)}$, hence grade $I\left(\varphi_{2}\right) \geqq$ grade $I\left(\varphi_{3}\right) \geqq 3$ (the latter by exactness of $\mathscr{C})$. Since, in any case, grade $J_{n-2}=$ grade $I\left(\varphi_{2}\right)$, the upshot is that grade $J_{n-2} \geqq 3$. Therefore, also grade $I_{n}(f) \geqq 3$ and grade $I_{n-2}\left(f^{\prime}\right) \geqq 3$.

In order to finish the proof of the implication (ii) $\Rightarrow$ (i), we claim that $\sqrt{I\left(\varphi_{4}\right)}=\sqrt{I_{n}(f)+I_{n-2}\left(f^{\prime}\right)}$. Again, by a specialization argument, it suffices to look at the generic situation. Let $P$ be a minimal prime of $I_{n}(f)+I_{n-2}\left(f^{\prime}\right)$ and assume $n \geqq 4$. Clearly, $I_{n-3}\left(f^{\prime}\right) \notin P$ (recall we are now dealing with the generic case). By the usual procedure of inverting a minor in $I_{n-3}\left(f^{\prime}\right)$ and applying elementary transformations to $f$ over $R[X]_{P}$, we reduce the question to verifying that $I\left(\varphi_{4}\right)$ is $P$-primary when $n=3$. But in this case, a straightforward computation yields directly that $\sqrt{I\left(\varphi_{4}\right)}=I_{3}(f)+I_{1}\left(f^{\prime}\right)$.

The claim about the homological dimension is standard and we leave to the reader the verification that it follows simply from the preceding arguments.

We close this section with some results about the analytic behaviour of the ideal $J$. We let $R:=k[X]$, where $k$ is a field and $X$ an $n \times(n+2)$ generic matrix. Set $J:=J_{n-2}(X)$. 
As before, $v($ ) will denote minimal number of generators. The analytic spread of an ideal $I$ in a local ring will be denoted by $l(I)$.

Proposition 3.4. (i) $v\left(J_{P}\right) \leqq \max \left\{\mathrm{ht}(P)-1\right.$, ht $\left.\left(J_{P}\right)\right\}$ for every prime ideal $P$ distinct from $I_{n}(\boldsymbol{X})+I_{n-2}\left(\boldsymbol{X}^{\prime}\right)$, while $v\left(J_{P}\right)=5$ for $P=I_{n}(\boldsymbol{X})+I_{n-2}\left(\boldsymbol{X}^{\prime}\right)$.

(ii) $l\left(J_{P}\right) \leqq \max \left\{\mathrm{ht}(P)-1, \mathrm{ht}\left(J_{P}\right)\right\}$.

P r o of. Since $J$ itself is generated by six elements, we may assume that ht $(P) \leqq 6$. We first prove (i): if $n \geqq 4$ then ht $\left(I_{1}\left(X^{\prime}\right)\right)=n(n-2) \geqq 8>$ ht $(P)$, so by inverting an entry of $\boldsymbol{X}^{\prime}$ and applying suitable elementary transformations to the resulting matrix, one reduces the question to the case $n=3$ - we leave out the case $n=2$ which is well-known to be a complete intersection in the punctured spectrum.

Now, for $n=3$ we may assume that $P \supset I_{1}\left(X^{\prime}\right)+I_{3}(X)$ as otherwise $J_{P}=I_{1}\left(X^{\prime}\right)_{P}$ or $J_{P}=I_{3}(X)_{P}$, which are well-known complete intersections (the latter because $P \neq I_{2}(X)$ as ht $\left(I_{2}(X)\right)>6$ ). Since even $P \ngtr I_{2}\left(X^{\prime \prime}\right)$, where $X^{\prime \prime}$ denotes the submatrix complementary to $X^{\prime}$ in $X$, we may further assume that the $2 \times 2$ minor $\delta$ corresponding to rows 1,2 and columns 2,3 does not belong to $P$. Inverting $\delta$ and applying suitable elementary transformations to $X$ over $R\left[\delta^{-1}\right]$, we obtain a matrix

$$
\boldsymbol{Y}:=\left[\begin{array}{ccccc}
Y_{1} & 1 & 0 & 0 & 0 \\
Y_{2} & 0 & 1 & 0 & 0 \\
Y_{3} & 0 & 0 & Y_{4} & Y_{5}
\end{array}\right],
$$

where $Y_{i},(i=1, \ldots, 5)$ are indeterminates in a new ring $\widetilde{R}$. But $J(Y)=J(X)_{\delta}$ as $Y_{1}, Y_{2}, Y_{3}$ are the result of elementary transformations applied solely to $X_{11}, X_{21}, X_{31}$. Therefore, $J(\boldsymbol{X})_{P}=J(\boldsymbol{Y})_{\tilde{P}}$, where $\widetilde{P}:=P \widetilde{R}$. A direct calculation shows that $J(\boldsymbol{Y})$ $=\left(Y_{1} Y_{4}, Y_{1} Y_{5}, Y_{2} Y_{4}, Y_{2} Y_{5}, Y_{3}\right)$, which proves our contention.

In order to prove (ii) it suffices, by (i), to show that $l\left(J_{P}\right) \leqq 4$ for the prime $P=I_{n}(X)+I_{n-2}\left(X^{\prime}\right)$. As above, after the standard identifications, we have $J(X)_{P}$ $\left.=\left(\left(Y_{1}, Y_{2}\right) \cap Y_{4}, Y_{5}\right)+\left(Y_{3}\right)\right)_{(Y)}$. The relation of analytic dependence given by $Y_{1} Y_{4} \cdot Y_{2} Y_{5}=Y_{1} Y_{5} \cdot Y_{2} Y_{4}$ shows that $l\left(J_{P}\right) \leqq 4$.

Corollary 3.5. With same notation as above, one has:

(i) $\operatorname{dim} S(J)=\operatorname{dim} R+1$ and $S(J)$ is not Cohen-Macaulay.

(ii) $\operatorname{dim} S\left(J / J^{2}\right)=\operatorname{dim} R$.

(iii) $\operatorname{gr}_{J}(R)$ is $R / J$-torsion free.

P r o of. (i) According to the formula of [13], the inequalities $v\left(J_{P}\right) \leqq$ ht $(P)+1$, for every prime $P$, already imply the required value for $\operatorname{dim} S(J)$. By [14], in this case, $S(J)$ cannot be Cohen-Macaulay unless it is a domain. However, the generators of $J$ are analytically dependent, a relation of dependence being given by straightening the product of the minors corresponding, respectively, to columns $1, \ldots, n-2, n-1, n+2$ and $1, \ldots, n-2, n, n+1$. 
(ii) This follows again from [13] and the earlier proposition.

(iii) From the estimates for the local analytic spreads given in the proposition and from [10], one has the required torsion-freeness. For a different proof, in greater generality, see [7].

The following points seemed to us worthwhile addressing:

1. Is there an "expected" free resolution of $S\left(J / J^{2}\right)$ over $R[\boldsymbol{T}]$ that comes from natural subcomplexes of (graded) Koszul complexes?

2. Is $S\left(J / J^{2}\right)$ Cohen-Macaulay?

The authors do not have the answer to the questions.

4. The complete intersection locus. For a noetherian ring $R$ and an ideal $I \subset R$, we consider the complete intersection locus of $I$. This is given by an open set $D(L) \subset \operatorname{Spec} R$ such that $P \in D(L)$ if and only if $I_{P}$ is generated by an $R_{P}$-sequence. Assuming some "regularity" condition, such ideal is easy to describe.

Lemma 4.1. Let $R$ be a Cohen-Macaulay noetherian ring and let $I \subset R$ be an ideal of finite homological dimension. If $F_{1} \stackrel{\varphi}{\longrightarrow} F_{0} \longrightarrow I \longrightarrow 0$ is a free presentation of $I$, then the open set $D\left(I_{\delta}(\varphi)\right) \subset \operatorname{Spec} R$ is the complete intersection locus of $I$, where $\delta$ stands for the deviation of $I:=\operatorname{rank}\left(F_{0}\right)-$ ht $(I)$.

P r o of. The argument can be transcribed from the proof of Corollary (1.2) in [27], by recalling that, in the presence of finite homological dimension, $I_{P}$ is a complete intersection if and only if $I_{P} / I_{P}^{2}$ is $R / P$-free.

The estimates for ht $(L)$ given in the literature ([8], [11], [27]), however sharp for some classes of ideals, are nearly inocuous in our present context. We shall derive the exact value of the codimension of the complete intersection locus, as well as a complete description of the generic components of Spec $R \backslash D(L)$, for the ideals studied in the earlier sections. Following the notation of [27], we set $c(I):=\operatorname{ht}(L)=\mathrm{ht}\left(I_{\delta}(\varphi)\right)$, where $\delta=$ deviation of $I$.

For completeness, we treat other cases of ideals of maximal minors fixing a set of columns. Only the generic case is considered, but presumably one could also deal with the general case as long as enough grade bounds are given.

Proposition 4.2. Let $R:=k[X]$ and let $J:=J_{n-1}(X) \subset R$ be the ideal generated by the maximal minors of the $n \times m$ matrix $X$ fixing the $n \times(n-1)$ initial submatrix $X^{\prime}$. Then:

(i) $\sqrt{I_{\delta}(\varphi)}=\ldots=\sqrt{I_{1}(\varphi)}=I_{n-2}\left(X^{\prime}\right) \cap I_{n}(X)$

(ii) $\mathcal{c}(J)=\min \{6, m-n+1\}$ if $n \geqq 3 ; c(J)=m-1$ if $n=2$.

Proof. (i) We refer to the presentation given in [1], to wit,

$$
A^{n+1} R^{m} \stackrel{\varphi}{\longrightarrow} A^{n-1} R^{n-1} \otimes A^{1} R^{m-n+1}=R^{m-n+1} \longrightarrow J \rightarrow 0,
$$


where $\varphi$ is the composition of the Cramer map $A^{n+1} R^{m} \rightarrow R^{m}$, given by

$$
w_{1} A \ldots \Lambda w_{n+1} \rightarrow \sum_{t}(-1)^{t}\left(\boldsymbol{X}\left(w_{1}\right) \Lambda \ldots A \widehat{X\left(w_{t}\right)} A \ldots A X\left(w_{n+1}\right)\right) w_{t},
$$

with the canonical projection $R^{m}=R^{n-1} \oplus R^{m-n+1} \rightarrow R^{m-n+1}$.

Now, obviously, $I_{1}(\varphi) \subset I_{n}(\boldsymbol{X})$. A closer inspection in the map $\varphi$ shows that also $I_{1}(\varphi) \subset I_{n-2}\left(X^{\prime}\right)$. Clearly, then $I_{\delta}(\varphi) \subset I_{n-2}\left(X^{\prime}\right) \cap I_{n}(X)$. Conversely, let $P \subset R$ be a prime such that $I_{\delta}(\varphi) \subset P$. Suppose $I_{n-2}\left(X^{\prime}\right) \neq P$. Then, by inverting a minor in $I_{n-2}\left(X^{\prime}\right)$ and performing suitable elementary transformations, one reduces the problem to the case where $n=2$. But, here it is easy to see that $I_{2}(X)=I_{1}(\varphi) \subset P$. This means that the original prime had to contain $I_{n}(X)$ if it did not contain $I_{n-2}\left(X^{\prime}\right)$. We must conclude that $\sqrt{I_{\delta}(\varphi)}=I_{n-2}\left(\boldsymbol{X}^{\prime}\right) \cap I_{n}(\boldsymbol{X})$. Of course this implies $\sqrt{I_{1}(\varphi)}=\ldots=\sqrt{I_{\delta}(\varphi)}$ $=I_{n-2}\left(X^{\prime}\right) \cap I_{n}(X)$ all the way through.

(ii) This is just reading heights in (i).

Proposition 4.3. Let $J:=J_{n-2}(X) \subset R:=k[X]$ be the ideal generated by the maximal minors of the $n \times(n+2)$ matrix $\boldsymbol{X}$, fixing the initial $n \times(n-2)$ submatrix $\boldsymbol{X}^{\prime}$. If $n \geqq 3$, one has:

(i) $\sqrt{I_{3}(\varphi)}=I_{n-3}\left(X^{\prime}\right) \cap I_{n-1}(X) \cap\left(I_{n-2}\left(X^{\prime}\right)+I_{n}(X)\right)$.

(ii) $c(J)=5$.

P r o of. We refer to the presentation of $J$ given in Section 3. A close reading of the map $\varphi$ in that presentation reveals that $I_{1}(\varphi) \subset I_{n-3}\left(X^{\prime}\right) \cap I_{n-1}(X)$. It is much subtler to see that $I_{3}(\varphi) \subset I_{n-2}\left(X^{\prime}\right)+I_{n}(X)$. Actually, we will prove a stronger statement, namely, that $I_{2}(\varphi) \subset I_{n-2}\left(X^{\prime}\right)+I_{n}(X)$. To see this, one writes the matrix of $\varphi$ in convenient bases, thus detecting two kinds of $2 \times 2$ minors: the ones whose terms contain a factor which is an $(n-1) \times(n-1)$ minor of $\boldsymbol{X}$ fixing $\boldsymbol{X}^{\prime}-$ these belong then to $I_{n-2}\left(\boldsymbol{X}^{\prime}\right)-$ and the ones containing terms whose factors do not all fix $\boldsymbol{X}^{\prime}$ - these can be expressed as sums of products of $(n-2) \times(n-2)$ minors of $X$ by $n \times n$ minors of $X$ (in the way of classical identities), hence belong to $I_{n}(X)$.

Summing up, we have $\sqrt{I_{3}(\varphi)} \subset I_{n-3}\left(X^{\prime}\right) \cap I_{n-1}(X) \cap\left(I_{n-2}\left(X^{\prime}\right)+I_{n}(X)\right)$. In order to prove equality, since $I_{3}(\varphi)$ defines the complete intersection locus of $J$, it is sufficient to show that $J$ is a complete intersection along those primes not containing any of the three above primes. Let $P$ be such a prime. If $n \geqq 4$, we may invert a minor belonging to $I_{n-3}\left(X^{\prime}\right)$ and perform elementary transformations on $X$, thus reducing the question to the case where $n=3$. Here we are assuming that either $I_{1}\left(X^{\prime}\right) \notin P$ or $I_{3}(X) \notin P$. Note, however, that $J=I_{1}\left(X^{\prime}\right) \cap I_{3}(X)$. On the other hand, $I_{1}\left(X^{\prime}\right)$ is even globally a complete intersection, while the complete intersection locus of $I_{3}(X)$ is given by $I_{2}(X)$. Since $P \notin I_{2}(X)$ as well, one is through.

(ii) This is reading heights above: ht $\left(I_{n-3}\left(X^{\prime}\right)\right)=8=\mathrm{ht}\left(I_{n-1}(X)\right)(n \geqq 4)$ and ht $\left(I_{n-2}\left(X^{\prime}\right)+I_{n}(X)\right)=5$ (cf., e.g., [12]). 
We close the section with the computation of the complete intersection locus of the presentation ideal of the associated graded ring studied in sections one and two.

Proposition 4.4. Let $X$ be an $n \times(n+2)$ matrix $(n \geqq 2)$ and let $I \subset R[T]$ be the presentation ideal of the associated graded ring of the ideal $J_{n-1}(X) \subset R:=k[X]$. Then:

(i) $\sqrt{I_{n-1}(\varphi)}=\left(I_{n-1}\left(X^{\prime}\right)+I_{n}(X \mid T)\right) \cap\left(I_{n-2}\left(X^{\prime}\right)+(T)\right)$,

(ii) $c(I)=6$.

Proof. We refer to the presentation of $I$ given in the earlier sections. As we saw there, the deviation of $I$ is $n-1$. From the presentation, one easily checks that $I_{n-1}(\varphi) \subset\left(I_{n-1}\left(X^{\prime}\right)\right.$ $\left.+I_{n}(X \mid T)\right) \cap\left(I_{n-2}\left(X^{\prime}\right)+(T)\right)$. Conversely, let $P \supset I$ be a prime ideal containing neither $I_{n-1}\left(X^{\prime}\right)$ $+I_{n}(X \mid \boldsymbol{T})$ nor $I_{n-2}\left(X^{\prime}\right)+(T)$. Note that $I=I_{n+1}(X \mid T)+J_{n-1}(X)$. Therefore, we must have $I_{n-3}\left(X^{\prime}\right) \notin P$. If $n \geqq 4$, invert a minor in $I_{n-3}\left(X^{\prime}\right)$, etc., so as to reduce the question to the case $n=3$. We are now given a prime $P \supset I$ such that $P$ does not contain either $I_{2}\left(X^{\prime}\right)+I_{3}(X \mid T)$ or $I_{1}\left(X^{\prime}\right)+(\boldsymbol{T})$. The case where $I_{1}\left(\boldsymbol{X}^{\prime}\right) \notin P$ can be further reduced to $n=2$; but, here an inspection shows that $I_{1}(\varphi)=I_{1}\left(X^{\prime}\right)+I_{2}(X \mid T)$ and we are through. Otherwise, let $I_{1}\left(X^{\prime}\right) \subset P$ (back to $n=3)$. In this case, we must have $(T) \notin P$ and $I_{3}\left(X^{\prime \prime} \mid T\right) \notin P$, where $X^{\prime \prime}$ is the complementary matrix of $X$ relative to $X^{\prime}$. One can check that, by inverting suitable elements and performing elementary transformations, the matrix $\boldsymbol{X} \mid \boldsymbol{T}$ can be brought to one of the following forms

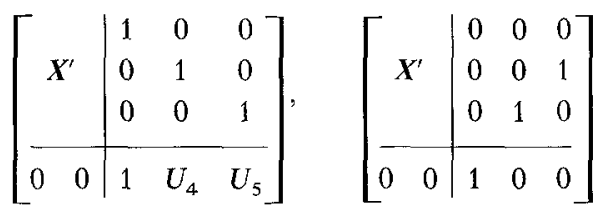

and, therefore, locally at $P, I$ is generated by the minors [23|12], [1345] and [2345].

(ii) Reading heights once more, one has:

ht $\left(I_{n-2}\left(X^{\prime}\right)+(T)\right)=6+3=9(n \geqq 3)$

ht $\left(I_{n-1}\left(X^{\prime}\right)+I_{n}(X \mid T)\right)=6$ (for this, note that $I_{n-1}\left(X^{\prime}\right)+I_{n}(X \mid T)$ is an associated prime of $\left.I_{n}(X \mid T)\right)$.

A side curiosity about the ideals considered in this work is that the codimension of their complete intersection locus is very nearly the minimum between their analytic spread and twice the codimension of their component of largest codimension - this is far better than the additive bound of [11].

\section{References}

[1] J. F. ANDRADE and A. Simis, A complex that resolves the ideal of minors having $n-1$ columns in common. Proc. Amer. Math. Soc. (2) 81, 217-219 (1981).

[2] J. F. ANDRADE and A. Simis, On ideals of minors fixing a submatrix. J. Algebra 102, 246-259 (1986).

[3] D. Buchsbaum and D. EIsenbud, Some structure theorems for finite free resolutions. Adv. in Math. 12, 84-139 (1974).

[4] D. BuCHSBAUM and D. EISENBUD, Algebra structure for finite free resolutions and some structure theorems for ideals of codimensions three. Amer. J. Math. 99, 447-485 (1977).

[5] D. Buchsbaum and D. S. Rim, A generalized Koszul complex. II. Depth and multiplicity. Trans. Amer. Math. Soc. 111, 197-224 (1964).

[6] W. BRuns and A. Srmis, Symmetric algebras of modules arising from a fixed submatrix of a generic matrix. J. Pure. Appl. Algebra 49, 227-245 (1987). 
[7] W. Bruns, A. Simis and N. V. Trung, Blow-ups of straigtening closed ideals in ordinal Hodge algebras. In preparation.

[8] G. Faltings, Ein Kriterium für vollständige Durchschnitte. Invent. Math. 62, 393-401 (1981).

[9] T. GUlliksen et $O$. NeGaRD, Un complex résolvant pour certain idéaux déterminantiels. C.R. Acad. Sci. Paris Sér. A 274, 16-19 (1972).

[10] C. HunEKE, On the associated graded ring of an ideal. Illinois J. Math. 26, 121-137 (1982).

[11] C. HunEkE, Criteria for complete intersections. J. London Math. Soc. 32, 19-30 (1985).

[12] M. Hochster and J. EAGON, Cohen-Macaulay rings, invariant theory and the generic perfection of determinantal loci. Amer. J. Math. 93, 1020-1058 (1971).

[13] C. Huneke and M. E. Rossi, The dimension and components of symmetric algebras. J. Algebra 98, 200-210 (1986).

[14] J. Herzog, A. Simis and W. Vasconcelos, Koszul homology and blowing-up rings. In: Lecture Notes Pure Appl. Math. 84, 79-169 (1981).

[15] J. Herzog, A. Simis and W. VASCONCELOS, "Most" reduced normal cones are quasi-Gorenstein. In preparation.

[16] C. Huneke and B. Ulrich, Residual Intersections. Preprint 1987.

[17] C. HunEke, A. Simis and W. VASCONCElos, Reduced normal cones are domains. Contemporary Math., to appear.

[18] J. Herzog and W. Vasconcelos, On the divisor class group of Rees algebras. J. Algebra 98, $182-188$ (1985).

[19] A. Kustrn, M. MILLER and B. ULRICH, Linkage theory of algebras with pure resolutions. J. Algebra 102, 199-228 (1986).

[20] C. Peskine et L. SzPIro, Dimension projective finie et cohomologie locale. Publications I.H.E.S. 42, 145-158 (1973).

[21] P. Pragacz and J. Weyman, Complexes associated with trace and evaluation; another approach to Lascoux's resolution. Adv. in Math. 57, 163-207 (1985).

[22] A. Simis, Multiplicities and Betti numbers of homogeneous ideals. In: Proceedings of the Curve Seminar, Rocca di Papa, Roma, 1985, LNM 1266, Berlin-Heidelberg-New York 1987.

[23] A. Simis and N. V. Trung, The divisor class group of ordinary and symbolic Rees algebras. Math. Z. 198, 479-491 (1988).

[24] A. SIMIS and W. VASCONCELOS, On the dimension and integrality of symmetric algebras. Math. Z. 177, 341-358 (1981).

[25] R. VillarreaL, Koszul homology of Cohen-Macaulay ideals. Ph. D. thesis, Rutgers University 1986.

[26] W. Vasconcelos, Koszul homology and the structure of low codimension Cohen-Macaulay ideals. Trans. Amer. Math. Soc. 301, 591-613 (1987).

[27] W. Vasconcelos, The complete intersection locus of certain ideals. J. Pure Appl. Algebra 38, $367-378$ (1985).

Anschrift der Autoren:

J. F. Andrade and A. Simis

Instituto de Matemática

Universidade Federal da Bahia

Av. Ademar de Barros, $\mathrm{s} / \mathrm{n}$

40210 Salvador, Bahia

Brazil 